\title{
AUDIODESKRIPTIONSÜBERSETZUNG ALS ALTERNATIVE METHODE DER FILMBESCHREIBUNG
}

\begin{abstract}
The translation of audio description as an alternative form of movie describing
This paper discusses the method of audio description as intralingustic and interlinguistic translation. It offers a contrastive analysis of the audio described scripts of the film Imagine of Andrzej Jakimowski in German and Polish, with a view on some of challenges that could arise when translating this type of text. After informations about creation of audio description and introduction of research in the field of the translation of existing audio descriptions, the paper suggests that similar conventions have evolved in German and Polish regarding both the content and expressions of ADs. These similarities are illustrated with reference to the German and Polish ADs for Imagine, which are analysed on the example of main characters and action places.
\end{abstract}

KEYWORDS: audio description, audio description as interlinguistic translation, guidelines for audio description, contrastive analysis of audio description

Die Medialität der Kultur und die voranschreitende Globalisierung gesellschaftlicher Prozesse im Zeitalter der rasanten Entwicklung der Informations- und Kommunikationstechnologie erfordern Neudefinitionen des Übersetzungsbegriffs und verlangen von der Translationswissenschaft Dynamik und Offenheit. Somit soll sich die Übersetzung nicht nur auf Prozesse beschränken, in denen aus einer Sprache in eine andere übertragen wird, sondern alle Vorgänge einschließen, in denen Kommunikationsbarrieren, seien sie sprachlicher (verschiedene Sprachen der Ausgangs- und Zielkultur) oder sensorischer Natur (auditive und visuelle Störungen), überwunden werden. Durch die Ermöglichung des Zugangs sensorisch beeinträchtigter Menschen zu Vorzügen und Möglichkeiten der Mediengesellschaft erhält der Übersetzungsbegriff eine entscheidende Erweiterung: Zugänglichkeit ist damit eine Form der Translation und Translation wiederum eine Form der Zugänglichkeit, die alle Bevölkerungsgruppen vereinigt und es ermöglicht, dass alle Menschen kulturelle Ereignisse, 
in weitestem Sinne des Wortes, genießen können (Díaz, Orero, Remael 2007: 13f.). Eine Ausprägung dieser Tendenz, die zahlreichen Medienformate auch körperlich eingeschränkten Personen zugänglich zu machen, ist die Audiodeskription, die von den praktizierenden Filmbeschreibern folgendermaßen definiert wird:

Der Begriff Audiodeskription meint ganz allgemein das hörbare Beschreiben von visuellen Eindrücken. Audiodeskription ist sozusagen eine akustische Untertitelung und bedeutet für das Medium Film, ihn mit zusätzlichen akustischen Bildbeschreibungen zu ergänzen. Visuelle Elemente wie Orte, Landschaften, Personen, Gestik, Kameraführung - alles, was zu sehen ist und was insbesondere für das Verständnis der Handlung und das ästhetische Erleben des Werkes wichtig ist, in Sprache umzusetzen. Der so entstandene Beschreibungstext ist in den Dialogpausen des Filmes zu hören. Auf diese Weise entsteht für den blinden und sehbehinderten Zuschauer ein ganzheitlicher Eindruck vom Filmgeschehen. Er erhält dadurch die Möglichkeit, sich das Medium Film ohne fremde Hilfe zugänglich zu machen und so am kulturellen Leben der Gesellschaft teilzunehmen. (Vereinigung Deutscher Filmbeschreiber: Audiodeskription)

Zwar ist die Audiodeskription, so Bernd Benecke (2014: 31), praktisch von zunehmender Bedeutung, wissenschaftlich allerdings noch nicht eingeordnet. In den meisten Untersuchungen wird sie in den translationswissenschaftlichen Rahmen mit einbezogen. Wenn man die Definition der Audiodeskription als „Reduktion eines komplexen multimedialen Textes auf einen nur über das Hören zu erfahrenden Text" (Fix 2005: 7) anführt, ergibt sich daraus eine Reihe von Bezügen zur übersetzungswissenschaftlichen Forschung. Als intermediale Umsetzung wird die Audiodeskription zum Gegenstand übersetzungswissenschaftlicher und audiovisueller Reflexion, was den interdisziplinären Charakter der Untersuchungen impliziert und zur Problematik der Filmbeschreibung unter den spezifischen Fragestellungen der Linguistik samt Bildlinguistik, Semiotik und Filmwissenschaft führt.

Werden die zentralen Fragestellungen des „In-Worte-Fassens von Bildinformationen für Blinde und Sehbehinderte" (Benecke 2014: Vorwort) unter dem Gesichtspunkt der genannten Disziplinen beleuchtet und wird Bezug auf den translatorischen Aspekt genommen, erfordert die Frage, ob es sich im jeweiligen Fall um eine intersemiotische oder interlinguale Übersetzung handelt, eine Präzisierung. Wird eine Audiodeskription neu erstellt, findet ein Wechsel der verwendeten Zeichen statt und es ergibt sich eine Form der intersemiotischen Übersetzung. Wird dagegen zunächst in der Ausgangssprache vom Bild zum Wort und der Text schließlich in die Zielsprache übertragen, so spricht man von der interlingualen Übersetzung. Die Übersetzung der Audiodeskription von einer Sprache in eine andere ist keine gängige Praxis. In der Regel fertigt man eine Filmbeschreibung für jede Sprache bzw. für jedes Land neu an. Selbst in den USA und in Großbritannien wird nicht die Version des jeweils anderen übernommen, sondern separat erstellt. Eine gewisse Reflexion zur komplexen Problematik des Themas hat sich bereits in der Literatur der audiovisuellen Übersetzung niedergeschlagen. Einen wichtigen Schritt in der Forschung der Audiodeskription als interlingualer Übersetzung stellen die Untersuchungen von 
Marleen Weißbach (vgl. 2012: 343-409) dar. In den Mittelpunkt ihrer Überlegungen stellt Weißbach die Frage, durch welche prototypischen Text- und Sprachgestaltungsmuster die Audiodeskription in Deutschland und Großbritannien gekennzeichnet ist und welche Schwierigkeiten sich daraus für die Übersetzung vom Englischen ins Deutsche ergeben. Durch das Aufzeigen von Gemeinsamkeiten und Unterschieden in der kontrastiven Analyse der englischen und deutschen Audiodeskription des Films Brokeback Mountain verdeutlicht sie, auf welche Aspekte ein Übersetzer achten muss, um landestypische Konventionen nicht zu übersehen und die Erwartungen der Adressaten angemessen zu erfüllen (vgl. Weißbach 2012: 347f.).

Der vorliegende Beitrag schließt an diese Untersuchungen an und möchte einen weiteren Baustein zu der übersetzungsrelevanten Reflexion über die Audiodeskription liefern. Den Mittelpunkt der Arbeit bildet eine kontrastive Analyse der deutschen und der polnischen Audiodeskription des Films Imagine von Andrzej Jakimowski. Im Vorfeld der Analyse werden zunächst unter besonderer Berücksichtigung der Reflexion polnischer Forscher und Praktiker auf diesem Gebiet theoretische Grundlagen zur Audiodeskription in Erinnerung gebracht, um der Diskussion in den zentralen Fragen der Filmbeschreibung neue Denkanstöße zu geben. Die präsentierten Untersuchungen legen viele weitere Problemfelder offen, die weit über den Rahmen des vorliegenden Beitrags hinausgehen. Der Aufsatz ist somit als Pilotstudie konzipiert und möchte als Anreiz und zugleich Ankündigung weiterer Untersuchungen auf diesem Gebiet dienen.

\title{
Audiodeskription - Entstehung und Wesen einer Textsorte
}

\begin{abstract}
Ich weiß es, ich weiß es! Ich habe die ganze Geschichte von Anfang bis Ende mit erlebt, kenne ihre Details, Nuancen, kann über all das erzählen, was ich nicht gesehen habe, was ich seit ein paar Jahren keine Chance zu sehen hatte, es gibt in meinen Gedanken Farbenspiel, es gibt Licht und wahre Bilder, dieselbe, die so stark Leute gerührt haben, die nebenan, hinter mir, um mich herum gesessen haben. Es ist schön, schön. [...] Ich zögre-zaudre ein bisschen, auszugehen, den Ort zu verlassen, der wieder Sinn gewonnen hat, der nah wurde, interessant, eine für mich verständliche Sprache spricht, die aufhörte, mit mir so irritierendes, manchmal schmerzliches Verschweigen, Geheimnisse und Täuschungen zu spielen. (Więckowski 2010: 5) ${ }^{1}$
\end{abstract}

Dieser enthusiastische Bericht über die Rezeption eines Hörfilms von Robert Więckowski, Chefredakteur der vom Polnischen Blindenverband herausgegebenen Monatszeitschrift Pochodnia, erschien vier Jahre nach der ersten Filmvorstellung mit Audiodeskription in Polen und mag eine Ausführung über die Rolle der Audiodeskription ersetzen. ${ }^{2}$ Am 27. Novemer 2006 wurde im Kino Pokój in Białystok, der

\footnotetext{
${ }^{1}$ Alle polnischen Zitate in der Übersetzung der Autorin des vorliegenden Beitrags.

${ }^{2}$ Zur Rolle der Audiodeskription u.a. bei Ulla Fix (2011: 307), Marleen Weißbach (2012: 350f.), Mateusz Ciborowski (2008: 136f.), Agnieszka Szarkowska (2008: 125-130), Deutsche Hörfilm GmbH, Vereinigung Deutscher Filmbeschreiber.
} 
Wiege der polnischen Audiodeskription, der Film Statyści von Michał Kwieciński vorgeführt. Die Idee der akustischen Bildbeschreibung hat natürlich in Familien und Gemeinschaften mit blinden und sehbehinderten Mitgliedern eine lange Tradition. Als professionelles Verfahren begann die Geschichte der akustischen Bildbeschreibung in den USA, nachdem Gregory Frazier der Frau eines blinden Freundes dabei zugehört hatte, wie sie ihrem Mann die Geschehnisse auf der Leinwand zuflüsterte. Seit den 1970er Jahren entwickelte Frazier dann an der San Francisco University systematisch seine Methode der akustischen Präsentation visueller Informationen, die erstmals 1989 auf den Filmfestspielen in Cannes vorgestellt wurde. Die Berichte über die Audiodeskription beim Filmfestival in Cannes führten 1989 in München zur Gründung der ersten Filmbeschreibergruppe und zum ersten beschriebenen Film - der US-Komödie Die Glücksjäger. In Polen wurde die Initiative der professionellen Herstellung von Audiodeskription von zwei blinden Personen ergriffen - von Barbara Szymańska und Tomasz Strzymiński. Sie gründeten die Stiftung Audiodeskrypcja, die sich zum Ziel setzt, das Verfahren der Audiodeskription, so Strzymiński (2008: 129), in den Medien zu verbreiten und das gesellschaftliche Bewusstsein dafür zu steigern, dass eine Sehbehinderung nicht bedeuten muss, keinen Zugang zur Bildkultur zu haben. Sie veranstalten Schulungen im Bereich der Audiodeskription und haben ein polnisches Regelwerk zur Erstellung der Audiodeskription erarbeitet.

Die Basis zugänglicher Hörfilme und Hörtheater in der polnischsprachigen Version findet man auf der Webseite der polnischen Stiftung Kultury bez barier (vgl. dazu: Kultury bez barier: Filmy). Sie umfasst zur Zeit ca. 90 Positionen, beispielsweise Filme in der Regie von Andrzej Wajda Człowiek z marmuru, Człowiek z żela$z a$, Ziemia obiecana, sowie die neuesten polnischen und ausländischen Produktionen wie Pokłosie in der Regie von Władysław Pasikowski, Róża von Wojciech Smarzowski oder Nakaches und Toledans Intouchables. Manche Filme wurden auch live in Kinos, u.a. im Rahmen der Polnischen Filmfestspiele in Gdynia, audiodeskribiert, z.B. Rysa von Michał Rosa, Świadek koronny von Sypniewski und Filipiak, Wesele in der Regie von Wojciech Smarzowski oder Krzysztof Zanussis Serce na dtoni (vgl. dazu: Chmiel, Szymańska 2011/2012: 279f.). Ein weiteres Film- und Fernsehserienangebot ist über die Internetplattform des öffentlichen Fernsehens Telewizja Polska S.A. zugänglich, u.a. TV-Serien wie Tajemnica twierdzy szyfrów, Determinator, Magiczne drzewo. Weiterhin herrscht zwar viel Nachholbedarf im Bereich blindengerechter Kulturveranstaltungen, besonders wenn man den Maßstab der Barrierefreiheit an Großbritannien oder Deutschland anlegt (vgl. dazu: Weißbach 2012: 352357), dennoch war die Vorführung des Films Statyści und die Gründung der Stiftung Audiodeskription ein Wendepunkt in der Entwicklung des Sichtbarmachens visueller Inhalte und zog sowohl auf der legislativen Ebene als auch in der akademischen Diskussion eine Reihe von weiteren Initiativen um die dringlichen Fragestellungen der Audiodeskription nach sich. Am 1. Juli 2011 trat das Rundfunk- und Fernsehge- 
setz in Kraft, das u.a. zum Ziel hat, die Benachteiligung von hör- und sehbehinderten Menschen zu beseitigen, und demgemäß sind die polnischen Sender verpflichtet, vierteljährlich mindestens $10 \%$ ihres Angebots durch Untertitel für Hörgeschädigte über Audiodeskription und Zeichensprache zugänglich zu machen. ${ }^{3}$

Nachdem die für die vorliegende Arbeit gewichtige Problematik angedeutet und vor dem Hintergrund der Entwicklung des Filmbeschreibungsverfahrens dargestellt worden ist, soll in den folgenden Ausführungen die zentrale Fragestellung der Audiodeskription genauer beleuchtet werden. Bei der Audiodeskription werden optische Informationen (z.B. in einem Film, einem Theaterstück, in einer Oper oder in einem Museum) zunächst in einen geschriebenen Text übertragen, der dann wiederum akustisch präsentiert wird. Blinde und sehbehinderte Menschen bekommen mithilfe dieses Verfahrens die für sie nicht erfassbaren Informationen im Bereich des Hörfilms, des Hörtheaters oder der Höroper vermittelt - vor allem Informationen zur Handlung, zum Aussehen der Figuren, zu deren Körpersprache und Gesichtsausdruck sowie zu Schauplätzen (vgl. dazu: Benecke 2014: 1). Aus der Fülle der Informationen, die bewegte Bilder dem Rezipienten bieten, können bei der „Reduktion eines komplexen multimedialen Textes auf einen nur über das Hören zu erfahrenden Text" (Fix 2005: 7) nicht alle Inhalte berücksichtigt werden, es muss eine Auswahl stattfinden. Diese Auswahl steht, wie Benecke betont (Benecke 2014: 2), unter der Einschränkung, dass die gewählten Informationen zwar einerseits im Zusammenspiel mit Dialogen, Geräuschen und Musik einen Sinn ergeben müssen, andererseits aber physisch nur den freien Raum zwischen Dialogen, Geräuschen und Musik nutzen können. Einerseits soll der Beschreiber in dem Prozess des Hörbarmachens der visuellen Inhalte so viele der vorhandenen Informationen wie möglich aufnehmen, damit Blinde und Sehbehinderte das Werk gut und umfassend erleben und genießen können, dabei darf aber die akustische Beschreibung die Rezeption des Tons samt den Dialogen oder Musikeffekten nicht beeinträchtigt werden. Damit ist die Frage der filmischen Poetik angesprochen. Der bewegte Bildstreifen bringt eine außergewöhnliche Komplexität der Bedeutungsherstellung mit sich. Die bildliche Diskursivität mit ihrer Polyphonie verschiedener Bewegungsformen bildet die Basis für die Auswahl weiterer Gestaltungsmittel. Film als Sprache verfügt über eine Reihe filmspezifischer und aus anderen Kunsttraditionen adaptierter Ausdrucksmittel, u.a. Sprache, Musik, Geräusche, Dekor, Kostüme, Schauspielkunst, Kameraverhalten und Montage. ${ }^{4}$ Dies macht die Vieldimensionalität des Films aus, der nicht nur als Ware der Filmindustrie und als kompliziertes Instrument des Geschichtenerzählens aufzufassen ist, sondern den man vor allem in den Kategorien der Kunst wahrneh-

\footnotetext{
${ }^{3} \mathrm{Zu}$ den Rechtsregelungen auf dem Gebiet der Barrierefreiheit in Bezug auf verschiedene Medienformate in Europa vgl. u.a. Łukasz Bogucki (Bogucki 2013: 22f.).

${ }^{4}$ Zur Analyse der Filmsprache im Kontext der audiovisuellen Übersetzung bei Małgorzata Korycińska-Wegner (2011).
} 
men soll. Die wichtige Rolle der Filmpoetik in der Filmbeschreiberarbeit charakterisiert Izabela Künstler, Redakteurin der Untertitel für Hörgeschädigte und Autorin zahlreicher Audiodeskriptionen für Hörfilme, Hörtheater- und Museen, wie folgt:

Erlebnis. Das erwarten wir im Kontakt mit der Kunst, unabhängig von der Art ihrer Wahrnehmung. Die Aufgabe des Audiodeskriptors ist solch einen Text zu verfassen, der verursacht, dass das Werk mit der Audiodeskription auch berühren wird. Dass die Rezipientenempfindungen, die das Werk mit den Ohren sehen, den Empfindungen der Zuschauer, die es sich mit den Augen ansehen, ähnlich werden. (Künstler 2014: 142)

Die bereits genannten Aspekte sind für die Spezifik der Audiodeskription vor dem Hintergrund anderer Formen der audiovisuellen Übersetzung entscheidend. Sie betonen zugleich die besondere Aufgabe des Filmbeschreibers ${ }^{5}$ als eines Metarezipienten des Filmtexts, der stets ,das Spannungsverhältnis von zur Verfügung stehender Zeit und Optimierung von Information, von Informationsvermittlung und Rezeptionssteuerung sowie von Beschreibung und Interpretation in einer sinnvollen Balance zu halten" (Kluckhohn 2005: 65) versucht. Der Beschreiber sieht sich bei der Bearbeitung ,akustischer Untertitel“ (Fix 2005: 8) stets mit den Fragen konfrontiert, mit welchen Wörtern und Sätzen konkrete Bildinhalte in einen schriftlichen Text umgewandelt werden und in welche Lücken zwischen den Toninformationen die ausgewählte Bildinformation eingefügt werden soll, um ein sinnvolles Ganzes zu bilden. Bezüglich der genannten Schwierigkeiten argumentiert die Linguistik damit, dass „eine Bildbedeutung nicht klar eingegrenzt werden kann. Bilder bieten dem Rezipienten vielmehr ein Bedeutungspotential, das durch einen Kontext aktiviert und erschlossen werden muss" (Stöckl 2011: 49). Damit wird die Frage der besonderen Herausforderungen an den Filmbeschreiber sowie seiner Kompetenzen angesprochen. Unter allen in der Diskussion um den Hörfilm genannten Kompetenzen tritt die Sorgfalt der Wortwahl in den Vordergrund. ${ }^{6}$

Die Diskussion um die für die Audiodeskription zentrale Fragestellung, wie man bildliche Darstellungen in sprachliche übertragen soll, wird im Spannungsfeld der sprachlichen „Nüchternheit“ und „Expressivität“ (Fix 2011: 312) geführt. Einerseits wird öfters gegen die Filmbeschreiber der Vorwurf erhoben, sich mit den bloßen

\footnotetext{
${ }^{5}$ Der Begriff des Beschreibers wird an dieser Stelle auf den Filmbeschreiber eingeschränkt - nicht nur im Hinblick auf das Thema des vorliegenden Beitrags, sondern auch in Hinsicht darauf, dass, wie Benecke zu Recht bemerkt, z.B. im Fall des Hörmuseums diese Einschränkung entfällt (vgl. Benecke 2014: 2).

${ }^{6}$ Die Zeit- und Raumbeschränkungen, die die Audiodeskription dem Filmbeschreiber auferlegt, führten zur Erscheinung der Idee einer Audioeinführung, die auf der Zusammenfassung wichtiger Informationen zum Hintergrund eines Films und seiner formalen Auffälligkeiten beruht, die sich die blinden oder sehbehinderten Rezipienten vorab im Internet anhören können bzw. die ihnen vor der Filmvorstellung in Blindenschrift oder durch Vorlesen zugänglich gemacht werden. Mehr dazu u.a. bei: Benecke (2014: 21), Masłowska (2014: 39-47).
} 
inhaltlichen Informationen zu begnügen, also z.B. lediglich „wer wo steht“, „wer wohin geht", „wie jemand aussieht" anzugeben (vgl. dazu: Fix 2011: 312), andererseits aber werden eine zu oberflächliche Verwendung der in den Guidelines definierten Regeln und Konventionen sowie eine zu subjektive Vermittlung des Bildinhalts und das Niederschreiben persönlicher Empfindungen als häufig begangene Fehler des Filmbeschreibers genannt (vgl. dazu: Szarkowska 2008: 127; Strzymiński/ Szymańska 2010: 4). Eine gewisse Diskrepanz in dieser Frage ist zwischen den in Form von Regeln bearbeiteten Standards und der Sichtweise der praktizierenden Filmbeschreiber zu beobachten, die eine Assoziation mit der Diskrepanz zwischen Theorie und Praxis nahe legt. Barbara Szymańska und Tomasz Strzymiński berufen sich im polnischen Regelwerk zur Audiodeskription auf Joel Snyder, Gründer von Audio Description Associates, der die Filmbeschreibung mit dem Haiku vergleicht durch ästhetische Schlichtheit, Präzision und große Wortkargheit ist jeder Haikutext ein Spiegelbild eines Weltausschnitts (vgl. dazu: Szymańska/Strzymiński 2010: 22). Krzysztof Szubzda, der erste polnische Filmbeschreiber, definiert dagegen die Audiodeskriptionsbearbeitung als ständiges Balancieren zwischen objektiver Informationsvermittlung und gewissen Andeutungen und Interpretationen zwecks Bildhaftigkeit und effektiver Informationsvermittlung (vgl. Szarkowska 2008: 133). Auch Izabela Künstler betont die Rolle der bildhaften Ausdrucksweise in Audiodeskriptionstexten: „Ich suche nach den besten Worten. Das beste Wort ist suggestiv, zutreffend, evoziert angemessene Assoziationen und Vorstellungen, erweckt keine Zweifel, schafft Atmosphäre“ (Künstler 2010: 14). Weiter führt sie aus: „Vor die Alternative gestellt, was zu lassen und worauf aus Zeitmangel zu verzichten ist - wähle ich das Wort aus, das die Atmosphäre betont" (Künstler 2010: 14). In der Diskussion um die Objektivität des „In-Worte-Fassens von Bildinformationen“ (Benecke 2014: Vorwort) macht Szubzda auf die sich aus den unterschiedlichen Bedürfnissen und Ansprüchen ergebenden Zuschauerpräferenzen aufmerksam, die vom Alter und Grad der Erblindung abhängig sind. Die Adressatengruppe von Audiodeskriptionstexten wird nicht über Aspekte wie Alter, Wissensstand oder soziale Herkunft definiert, das einzige Kriterium bildet die visuelle Einschränkung der Rezipienten. Doch wie Szubzda berichtet, finden die jüngeren Zuschauer die Narration oft überfüllt, die älteren dagegen fordern mehr Details. Hörgeschädigte wissen bestimmte Andeutungen, Interpretation zu schätzen, die von den Blinden als ,Wortwörtlichkeit und idiotensichere Erklärungen“ bewertet werden (Szarkowska 2008: 133).

Die bereits angeführten Untersuchungen betonen die vom Filmbeschreiber geforderten Kompetenzen. Abgesehen von einer gewissen Affinität zum Filmschaffen und zur Poetik der Filmsprache sowie vom nötigen Wissen um die Grundsätze der Audiodeskription, fällt den Sprachkompetenzen eine besondere Rolle zu, die nicht nur als sprachliche Korrektheit und Reichtum der Sprache verstanden werden, sondern im Zusammenhang mit dem mentalen Bildbegriff und der damit verbundenen sprachlichen Anschaulichkeit und Bildhaftigkeit zu bedenken sind: 
Audiodeskriptoren müssen wissen, welche Vorstellungsinhalte, welche mentalen Bilder an welche Wörter und Wendungen gebunden sind. Welche Vorstellung hat die Sprachgemeinschaft und hat speziell der nichtsehende Mensch, wenn z.B. die Rede von einer jungen Frau ist. Gehören zu dem mentalen Bild, das der Betreffende hat, wenn er den Ausdruck hört, auch schlank und sportlich? Oder muss das explizit gesagt werden? (Fix 2011:310)

Trotz aller Ausführungen scheint die Frage nach der Möglichkeit einer Festlegung sprachlicher Adäquatheit nach wie vor unbeantwortet zu sein. Die Komplexität der Verhältnisse im Spannungsfeld sprachlicher Nüchternheit und Expressivität in der Audiodeskription fasst diese Bemerkung von Künstler zutreffend zusammen: „Kann eine Audiodeskription objektiv sein? Ja! Sie ist genauso objektiv wie jedes Werk und genauso subjektiv wie seine Wahrnehmung“ (Künstler 2014: 151).

\section{Zur Forschungslage}

Wie Benecke bemerkt (2014: 23), ist die Aufstellung von Regelwerken für die weltweite Professionalisierung der Audiodeskription für die Praxis im 21. Jahrhundert kennzeichnend. Diese Guidelines legen allgemeine Grundsätze für die akustische Bildbeschreibung fest und bilden einen Großteil der Literatur zur Audiodeskription. Eines der ersten Regelwerke war die im Jahre 2000 in Großbritannien erschienene ITC Guidance in Standards for Audio Description. In Deutschland gilt das 1997 formulierte und 2004 von den Filmbeschreibern Bernd Benecke und Elmar Dosch vom Bayerischen Rundfunk überarbeitete Regelwerk Wenn aus Bildern Worte werden - Durch Audio-Description zum Hörfilm, in dem sie neben den Informationen zu Geschichte und Produktion von Hörfilmen auch erklären, worauf bei der Filmbeschreibung zu achten ist. Eine hilfreiche Zusammenfassung der wichtigsten Aspekte findet man auf der Webseite der Vereinigung Deutscher Filmbeschreiber. Wie bereits erwähnt, veröffentlichten Barbara Szymańska und Tomasz Strzymiński 2010 in Białystok das polnische Regelwerk zur Erstellung der Audiodeskription für audiovisuelle Darbietungen Obraz stowem malowany [Das Bild mit Worten gemalt]. Der polnische Leitfaden setzt sich aus vier Kapiteln zusammen: Einführung, Audiodeskription der audiovisuellen Produktionen, Was und wie beschreiben sowie Aufnahme des Manuskripts im Tonstudio. Die Einführung liefert Definitionen der grundlegenden Begriffe sowie die wichtigsten Informationen zur Geschichte der Filmbeschreibung sowie einige Bemerkungen zu Rezeption und Zuschauerpräferenzen. In Kapitel zwei folgen allgemeine inhaltliche und formale Regeln der Hörfilmbearbeitung sowie die Etappen der Entstehung eines Hörfilms. Kapitel drei liefert detaillierte Informationen zu den inhaltlichen und sprachlichen Standards der Filmbeschreiberarbeit, u.a. die Einführung von Charakteren, Beschreibung von Handlungsräumen, Figurencharakteristik, Gestik und Mimik der Figuren, Farben, Soziolekte, Syntax, Verben und Umsetzung filmtechnischer Mittel. 
Mit den Guidelines wurde ,ein kollektiv geltender Kanon für professionelles, regelgebundenes Handeln vorgelegt" (Benecke 2014: 23), dennoch darf nicht übersehen werden, dass die Regelwerke von Land zu Land variieren, indem sie die Schulen und Traditionen der Manuskripterstellung und Zuschauerpräferenzen des jeweiligen Landes berücksichtigen. Für die Erstellung eines einheitlichen Leitfadens zur Filmbeschreibung plädiert Gert Vercauteren. Er skizziert eine europäische Guideline, die Regelungen zur Erstellung der Audiodeskription, Hinweise zur Aufnahme und Technik, gesetzliche Regelungen sowie Richtlinien für die Übersetzung der bereits vorhandenen Audiodeskriptionstexte beinhalten soll. Dies hätte nicht nur die Ausweitung des Verfahrens der akustischen Filmbeschreibung zur Folge, sondern würde auch eine hohe Qualität von Audiodeskriptionstexten gewährleisten (vgl. dazu: Vercauteren 2007: 138-149). Die Analyseergebnisse von Vercauteren stellen eine Skizze des vorgeschlagenen Regelwerks dar und können nur als - allerdings sehr aufschlussreiche - Indizien und Hinweise darauf genommen werden, wie man die Untersuchung fortsetzen sollte und welche Fragestellungen weiterer Erwägungen bedürfen. ${ }^{7}$

Wie bereits erwähnt, ist die Übersetzung der Audiodeskription keine gängige Praxis. Hörfilmfassungen werden in der Regel in jedem Land bzw. für jede Sprache separat erstellt. ${ }^{8}$ Julian Bourne und Catalina Jiménez Hurtado führten eine kontrastive Analyse der englischen und spanischen Audiodeskription des Films The Hours durch. ${ }^{9}$ Die Untersuchung hebt die für spanische Hörfilmfassungen charakteristischen Eigenschaften hervor, wie eine gewisse Wortkargheit in der Detailbeschreibung, familiären Wortschatz und kurze, einfache Sätze, die in der Opposition zu Beschreibungen stehen, an die die englischen Zuschauer gewöhnt sind (vgl. dazu: Bourne, Hurtado 2007: 184-186). Zuschauerpräferenzen und unterschiedliche Filmbeschreibungstraditionen gewinnen nach Bourne und Hurtado in der Diskussion um die Translation der Audiodeskription an Bedeutung. Wie beide jedoch bemerken (2007: 185), ist die Adaptation des Ausgangstextes hinsichtlich des entsprechenden

\footnotetext{
${ }^{7}$ An die Untersuchungen von Vercauteren knüpft Hansjörg Bittner an, und führt eine kontrastive Analyse der Audiodeskriptionsregelwerke aus Australien, Frankreich, Deutschland, Irland, Großbritannien und den USA durch (vgl. Bittner 2012: 41-61).

${ }^{8}$ Erwähnenswert sind an dieser Stelle die Untersuchungen der polnischen Forschergruppe AVT Lab, die im Rahmen des Projekts Audiodeskription zu Auslandsfilmen das Interesse sehbehinderter und blinder Zuschauer an fremdsprachigen Produktionen hervorgehoben und Lösungen bearbeitet hat, die bei der Bearbeitung von Audiodeskriptionen für ausländische Produktionen behilflich sein können. Aus den Untersuchungen geht hervor, dass in einer optimalen Audiodeskription zum fremdsprachigen Film das Voice Over-Verfahren eingesetzt werden soll und dass eine Audioeinführung empfehlenswert wäre (vgl. dazu: AVT Lab Audiovisual Translation Research Lab: Audiodeskrypcja do filmów zagranicznych).

${ }^{9}$ Eine Analyse polnischer Audiodeskriptionstexte vor dem Hintergrund der englischen Standards (ITC Guidance) findet man bei Chmiel und Mazur (2011, 2012: 279-296), jedoch ohne Besprechung der Implikationen für den Übersetzungsprozess.
} 
Wortschatzes und der Satzstrukturen an die Erwartungen des Rezipienten bei vielen Textsorten, wie zum Beispiel bei Reiseführern, Beipackzetteln oder wissenschaftlichen Beiträgen ein Bestandteil des Übersetzungsprozesses aus dem Englischen ins Spanische. Es stellt sich aber die Frage, ob eine solche Anpassung an die zielsprachlichen Regeln und Konventionen bei einem Kunstwerk in Frage kommt und ob man tatsächlich von diesem Verfahren in vielerlei Hinsicht profitieren kann.

Befürworter der Übersetzung der Audiodeskription errechnen, dass dieses Vorgehen den Vorteil der Einsparung von Zeit und Kosten hätte. Zugleich würde sie auch die Möglichkeit bieten, das Hörfilmangebot im jeweiligen Land zu erweitern (vgl. dazu: Bourne, Hurtado 2007: 176; Weißbach 2012: 367f.). In Europa werden Hörfilme u.a. auch in Belgien, Frankreich und Spanien produziert. Durch die Übersetzung bereits angefertigter, fremdsprachiger Filme könnte das Angebot für polnische Rezipienten erheblich erweitert und abwechslungsreicher gestaltet werden. Zudem könnten, wie Weißbach bemerkt (Weißbach 2012: 368), Länder, in denen bisher keine eigene Audiodeskriptionskultur gewachsen ist, von der Vorarbeit anderer Länder profitieren. Nach Veronika Hyks (2005: 6f.), einer der ersten Filmbeschreiberinnen in Großbritannien, stellt die Manuskriptübersetzung in Hinsicht auf Zeit- und Kosteneinsparung sowie größere Zugänglichkeit des Hörfilms zwar eine viel versprechende Perspektive dar, allerdings könne der Übersetzungsprozess und die Adaptation des Manuskripts mehr Zeit als der Schreibprozess von Grund auf in Anspruch nehmen. Die Gründe dieser Sachlage sieht sie in den unterschiedlichen Herausforderungen, die an die Kompetenzen eines Übersetzers und eines Filmbeschreibers gestellt werden. Während der Übersetzer treu, so Hyks (2005: 7), aus einer Sprache in eine andere überträgt, muss der Audiodeskriptor die wichtigsten visuellen Informationen auswählen und sie verbal vermitteln. In ihren Ausführungen gegen eine Audiodeskriptionsübersetzung weist sie auch auf die Unterschiede zwischen den Sprachen als Spiegelbild der jeweiligen Kultur hin (vgl. Hyks 2005: 6). Eine solche Auffassung der Problematik würde den Sinn der Übersetzung in Frage stellen und kann, wie Anna Jankowska (2014: 27) zu Recht bemerkt, aus einer mangelnden Kenntnis der Theorie und Praxis der Übersetzungskunst resultieren.

Den Sinn von Manuskriptübersetzungen ziehen auch Agnieszka Chmiel und Iwona Mazur in Zweifel. Sie berichten über die von Krzysztof Szubzda bei der Audiodeskription für die polnische Synchronfassung des Films Ice Age 2: The Meltdown gemachten Erfahrungen. Danach gefragt, bis zu welchem Grad ihm das bereits vorhandene Audiodeskriptionsskript für die englische Version des Films behilflich war, antwortet Szubzda, das es unbedeutend war. Er betont vor allem die unterschiedlichen Zuschauerpräferenzen, u.a. in Bezug auf die Metaphern- und Vergleichsverwendung (z.B. geläufiger Vergleich a building as tall as ten elephants placed on top of one another), die bei den polnischen Zuschauern, so Szubzda, nicht erwünscht sind (vgl. Chmiel, Mazur 2011, 2012: 282). 
Mit der Frage der Zeiteinsparung bei der Übersetzung der Audiodeskriptionsmanuskripte setzten sich polnische Forscher auseinander. 2014 präsentierte Anna Jankowska in ihrem Beitrag Thumaczenie jako alternatywna metoda tworzenia audiodeskrypcji (Jankowska 2014: 23-38) die Ergebnisse eines dreistufigen Experiments, an dem Studenten vom UNESCO Lehrstuhl für Übersetzung und Internationale Kommunikation der Jagiellonen-Universität, Studenten des Instituts für Englische Philologie sowie Absolventen des Masterstudiums im Bereich der audiovisuellen Übersetzung aus Imperial College in London teilnahmen. Im Rahmen der Untersuchung wurden die Audiodeskriptionen zu Synchronfassungen der Filme Harry Potter and the Philosopher's Stone, Harry Potter and the Prisoner of Azkaban, Ice Age: The Meltdown aus dem Englischen ins Polnische übertragen. Darüber hinaus wurde auch der Zeitaufwand bei der Übertragung der Manuskripte zu den englischsprachigen Filmen mit der spanischen Synchronfassung untersucht sowie der Zeitaufwand für die Verfassung und Übersetzung der Audiodeskription zu den englischsprachigen Produktionen, u.a. Mission Impossible, The Shawshank Redemption, für die polnischen Versionen mit Voice Over. Die Ergebnisse des Experiments haben bewiesen, dass die Bearbeitung der Audiodeskription durch die Übersetzung um das Dreifache weniger zeitaufwendig ist als die Filmbeschreibung, auch wenn sie von angehenden Audiodeskriptoren und Übersetzern bearbeitet wird (Jankowska 2014: 37f.). Einerseits kann das Anlegen des Zeit- und Kostenmaßstabs an ein Kunstwerk wundern und Widerspruch erregen, andererseits aber darf nicht vergessen werden, dass ein Film nicht nur in Kategorien der Kunst, sondern auch als Ware aufzufassen ist, die als Produkt der Filmindustrie einer immensen Kostenplanung unterliegt.

Einen bedeutenden Schritt in der Forschung zur Audiodeskriptionsübersetzung stellen die bereits angeführten Untersuchungen von Marleen Weißbach dar. Gemäß der Zusammenfassung ihrer Arbeitsergebnisse (Weißbach 2012: 403) konnten durch die kontrastive Analyse die Stellen aufgezeigt werden, an denen von deutschen und britischen Beschreibern unterschiedliche Vorgehensweisen gewählt wurden. Das Wissen um solche Unterschiede lässt die Audiodeskriptionsfassung als Übersetzungsvorlage an die Zielkultur anpassen und dank dessen kann man eine Übersetzung anfertigen, die mit den Erwartungen der Zuschauer im Einklang steht.

\section{Kontrastive Analyse der deutschen und der polnischen Audiodeskription}

In der kontrastiven Analyse der deutschen und polnischen Audiodeskriptionsfassung des Film Imagine soll gezeigt werden, in welchen Punkten die deutschen und die polnischen Guidelines zur Audiodeskriptionsgestaltung unter dem Gesichtspunkt des potentiellen Translationsprozesses voneinander abweichen und in welchen Punkten sie übereinstimmen. 


\section{Korpus}

Imagine $^{10}$ stellt bisher den einzigen Film dar, der sowohl im Deutschen als auch im Polnischen mit einer Audiodeskription versehen wurde und als DVD erhältlich ist. Der Grund dafür liegt darin, dass hierzulande, ähnlich wie in Deutschland (vgl. Weißbach 2012: 372), am häufigsten muttersprachliche Produktionen blindengerecht bearbeitet werden, gleichzeitig erscheinen nicht alle Hörfilmproduktionen auf DVD, sondern werden oftmals für Fernsehen, Kino bzw. Filmfestspiele erstellt. Imagine ist ein französisch-polnisch-portugiesisch-britisches Drama aus dem Jahre 2012 in der Regie von Andrzej Jakimowski. An dieser Stelle mag es erlaubt sein, den Filmemachern das Wort zu erteilen, die ihr Werk so darstellen:

Ian (Edward Hogg) ist blind und verfügt über eine besondere Fähigkeit: Über viele Jahre lernte er, seine Umwelt quasi mit den Ohren zu sehen. Durch genaues Zuhören orientiert er sich mit Hilfe von Schallwellen. Er wird nach Lissabon an eine weltbekannte, doch konservative Augenklinik gerufen, wo er verbesserte Techniken im Alltag von Blinden vermitteln soll. Doch was Ian seinen jungen Schülern eröffnet, ist weniger ein Orientierungskurs als eine völlig neue Art, in der Welt zu sein und das unlösbare Rätsel unserer Gegenwart durch Imagination und Neugier mit Sinn zu füllen. Ian verblüfft die Jugendlichen mit seinen aufregenden und riskanten Methoden. Sie sollen helfen, die so verbindliche Grenze zwischen Sehen und Nichtsehen zu überwinden. Doch das ist mitunter lebensgefährlich. Unter den Patienten ist auch die junge und scheue Eva (Alexandra Maria Lara), die spürt, dass ihr Ian etwas wiedergeben kann, das sie längst verloren hatte. Doch schnell gerät Ian unter den Verdacht, ein trügerisches Bild von der Welt vermittelt und damit das Leben seiner Schüler aufs Spiel gesetzt zu haben. Als er schließlich gezwungen wird, die Klinik zu verlassen, müssen Eva und die anderen Kursteilnehmer auf eigene Faust herausfinden, was die Imagination zur Wahrheit macht. Voller philosophischer Tiefe und mit einem souveränen Gespür für Komik lässt uns Imagine das Kino mit neuer Kraft sehen und hören. Mit poetischen Bildern und einer genauen Aufmerksamkeit für kleinste Geräusche zieht Regisseur Andrzej Jakimowski das Publikum in seine Geschichte von zwei Menschen hinein, die sich verlieben, ohne einander jemals gesehen zu haben. (Offizielle deutschsprachige Seite zum Film: Der Film)

Die Transkription der polnischen Beschreibung wurde für die vorliegende Untersuchung von den polnischen Filmbeschreiberinnen Monika Marciniak und Barbara Szymańska zur Verfügung gestellt. Die deutsche Audiodeskription wurde vom Unternehmen Eurotape Media Services GmbH angefertigt. Die Transkription zur deutschsprachigen Version wurde von der Verfasserin des vorliegenden Beitrags erstellt.

${ }^{10}$ Imagine: Polen/Frankreich/Portugal/Großbritannien 2012. Drehbuch: Andrzej Jakimowski. Regie: Andrzej Jakimowski. Musik: Tomasz Gąssowski. Kamera: Adam Bajerski. Schnitt: Cezary Grzesiuk. 


\title{
Analyse
}

\section{Handelnde Personen}

Das innere, dramaturgische Zentrum der filmischen Kreation bildet das Thema. Das Thema beschreibt den Konflikt, den der Protagonist zu lösen hat. Die Schilderung eines Problemlösungsprozesses setzt eine Figurenkonzeption voraus, bei der das von den Protagonisten verfolgte Ziel sowie ihre Charaktereigenschaften deutlich gezeigt werden. Über die Figuren erfolgt die Bestimmung des Verhältnisses von Nähe und Distanz seitens des Zuschauers zum Geschehen auf der Leinwand und seine Entwicklung vom Beobachter zum Teilnehmer der Geschichte, da er sich mit ihren Emotionen identifiziert. Als Beweger der Handlung und als Gestalten, die sich mit einem Konflikt auseinandersetzten müssen, sollten die Protagonisten als lebensechte Menschen geschildert werden (vgl. Korycińska-Wegner 2011: 23f.). Für sensorisch beeinträchtigte Zuschauer ist zudem die Information von Belang, wer gerade, besonders in Szenen ohne Dialog, auf dem Bildschirm agiert. In den deutschen Standards wird folgendes empfohlen:

\begin{abstract}
Der Zeitpunkt der Namensnennung innerhalb eines Films ist problematisch. Um nicht mehr Informationen zu geben, als auch der Sehende zur Verfügung hat, ist es nötig, die handelnden Personen über Charakteristika oder Eindrücke zu beschreiben. So wird eine Figur so lange „der Dunkelhaarige“ oder ,die mollige Frau“ genannt, bis sie im Film ihren Namen erhält. Erst dann kann das Filmbeschreiberteam den Namen aufgreifen und ihn für die weitere Beschreibung verwenden. Für die Namensnennung wird eine Stelle im Film ausgesucht, die es ermöglicht, noch weitere Informationen über die Hauptfigur wie Alter, Statur, Haarfarbe, Gesichtszüge, Augenfarbe etc. unterzubringen. Hierbei ist es besonders wichtig, das für den Film Wesentliche einer Person zu erfassen und zu veranschaulichen. (Vereinigung Deutscher Filmbeschreiber: Audiodeskription-HörfilmentstehungFilmbeschreibung-Handelnde Personen)
\end{abstract}

Auch in dem in Polen geltenden Regelwerk findet man den Hinweis, dass die Protagonisten ihren Namen erst bekommen, wenn er im Film gefallen ist. Bis dahin verwendet man kurze Beschreibungen, die charakteristische Eigenschaften vermitteln. Eine Ausnahme bilden hier die Protagonisten, deren Identität aus dramaturgischen Gründen verborgen bleibt.

Die Namensnennung wird in der vorliegenden Analyse am Beispiel der Hauptprotagonisten von Imagine - Ian und Eva besprochen. Ian ist blind und verfügt über eine besondere Fähigkeit: Durch genaues Zuhören orientiert er sich in seiner Umwelt mit Hilfe von Schallwellen. Er wird, wie bereits erwähnt, nach Lissabon an eine weltbekannte, jedoch konservative Augenklinik gerufen, wo er den Blinden bessere Techniken in der Bewältigung des Alltags vermitteln soll. Bevor der Name des Mannes genannt wird, begegnet er dem deutschen Rezipienten als ein Mann mit Sonnenbrille, der Besucher, der Gast. Noch in der Exposition des Films werden Stellen ausgesucht, die es ermöglichen, weitere Informationen über den Mann unter- 
zubringen: Durch ein großes Tor betritt der Mann ein Gebäude. Er hat braunes, gewelltes Haar, Koteletten und einen-paar-Tage-Bart. Zur Orientierung nutzt er Echoortung und lehnt den Blindenlangstock ab, deshalb hat er oft Schürfwunden im Gesicht. Dies wird auch in der Charakteristik der Figur wiedergegeben, bevor ihr Name eingeführt wird: Ein Pflaster klebt an seiner Stirn, neben dem Pflaster hat er frische Narbe. Als die Zuschauer aus den Dialogen erfahren, dass er gekommen ist, um an einer Privatklinik hinter Klostermauern den Unterricht für eine Klasse blinder Jugendlicher zu übernehmen, wird er zum Lehrer. Auch seine Kleidung wird in der Beschreibung berücksichtigt - Er trägt eine schwarze Lederjacke. Erst von dem Moment an, als der Klinikleiter sich an ihn wendet und ihn bei seinem Vornamen nennt - Ian, komm, bitte, komm her! $(0: 26: 47)$, agiert er in der Filmbeschreibung als Ian.

In der polnischen Audiodeskriptionsfassung wird Ian auch als Mann (mężczyzna) dargestellt. In den weiteren Minuten des Films erhält der Zuschauer Informationen zu seinem Aussehen. Die Filmbeschreiberinnen weisen auf die schwarze Jacke und Sonnenbrille - Mężczyzna w czarnej skórzanej kurtce i ciemnych okularach, Pflaster - Na tuku brwiowym mężczyzny plaster und auf den leichten Bart hin - Na twarzy lekki zarost. Bis zu dem Moment, in dem im Film sein Vorname gefallen ist, agiert Jan als mężczyzna und wird durch die den genannten Attributen inhärenten Informationen kennzeichnet. Es erscheint auch die Information über seine Augenfarbe, er wird als błękitnooki mężczyzna bezeichnet.

Als Ian als neuer Lehrer in der Klinik eintrifft, wird er gefragt, ob er lieber ein ruhiges Zimmer mit Blick auf eine Mauer, oder ein lautes Zimmer mit schönem Blick auf den Innenhof haben möchte. Er entscheidet sich für den Innenhof und wird damit Nachbar von Eva. Seine Aufgabe in den nächsten Wochen besteht darin, blinden Patienten eine neue Art der Orientierung ohne Blindenstock beizubringen. Die Schüler sind skeptisch und zugleich sehr gespannt. $\mathrm{Zu}$ ihnen gehört auch die scheue Eva, die sehr unter ihrer Behinderung leidet. Bevor die Hauptprotagonistin bei ihrem Vornamen genannt wird, lernen sie die deutschen Zuschauer als die Frau kennen. In den zwischen den Dialogen verbleibenden Lücken wird erst in dem Moment, als Ian sie bei ihrem Vornamen nennt - Und was ist mit dir, Eva? $(0: 34: 23)$, der Name in den Beschreibungen aufgegriffen. Zuvor werden zur Kennzeichnung von Eva durchgängig die Formulierungen die Frau, die junge Frau, die Frau am Fenster, die Nachbarin, die junge Nachbarin eingesetzt. In der Zwischenzeit erfährt der Zuschauer allmählich in den verbleibenden Lücken, dass sie Anfang 30 ist, schulterlanges, dunkelbraunes Haar und ein ernstes Gesicht hat, dass ihre großen, braunen Augen starr nach vorne gerichtet sind und dass sie ein kurzes, geblümtes Kleid und Flip-Flops trägt.

In der polnischen Audiodeskription werden die Hauptprotagonisten auch erst mit Vornamen versehen, nachdem diese im Filmdialog gefallen sind. Vorher werden Ian und Eva über Charakteristika wie Statur, Haarfarbe, Augenfarbe, Kleidung und 
Narben beschrieben - Ian: mężczyzna, mężczyzna w czarnej kurtce i ciemnych okularach, na tuku brwiowym mężczyzny plaster, na twarzy lekki zarost, błękitnooki mężczyzna; Eva: kobieta, kobieta w oknie, brazowe oczy i kasztanowe whosy do ramion, kobieta o kasztanowych włosach, przy ścianie zgrabne, kobiece nogi, sukienka odstania szczupte uda, na stopach japonki. Es werden keine Informationen über das Alter der Figuren gegeben.

\section{Handlungsräume}

Neben den Figuren stellt der Handlungsraum ein wichtiges Element der filmischen Dramaturgie dar und ist eines der Ausdrucksmittel der filmischen Poetik. Es unterliegt keinem Zweifel, dass es, wie Anja Seiffert (2005: 67) bemerkt, kein zufälliger Ereignisort ist, sondern vielmehr im Kontext des Films mit Sinn aufgeladen wird. Er betont in ganz wesentlichem Maße die Stimmung der jeweiligen Szene und tritt in Korrespondenz zum Charakter des Handlungsmoments und zu der Innenwelt der handelnden Personen.

Sowohl in den deutschen als auch polnischen Guidelines betont man, dass die Beschreibungen der Handlungsräume sachlich und neutral gehalten werden sollen. Im Vordergrund steht der allgemeine Eindruck des Ortes, und wenn die Zeit es zulässt, können auch einige Details, die darin enthalten sind, erwähnt werden, um einen konkreteren Eindruck von dem Ort zu geben.

Die Handlung von Imagine spielt größtenteils innerhalb der dicken Steinmauern der Klinik, was einen klaustrophobischen Effekt erweckt. Der Zuschauer kann die Enge des Raumes fühlen, die den Blick nicht weiter schweifen lässt. Für die Analyse der Darstellung von Handlungsorten in den beiden Audiodeskriptionsfassungen wurde stellvertretend der Hof der Klinik ausgewählt:

\footnotetext{
Die beiden durchqueren einen weiten Hof. Der Mann mit der Sonnenbrille schaut auf. Er erblickt die Tauben, die über niedrigen weißen Gebäuden flattern. Die Dächer sind mit Terrakottaziegeln gedeckt. Die Fenster sind vergittert. Ein Mädchen mit Brille öffnet ein Fenster. Neben ihr eine Kleine mit weißem Haarclip und geschlossenen Augen. Der Besucher hebt den Kopf. Ein Mädchen mit Stirnband tritt an ein Fenstergitter. Der Gast lächelt. Ein Pflaster klebt an seiner Stirn. Hinter einem anderen Fenstergitter schauen ein kleiner Junge und dunkelhäutiger Jugendlicher in den Hof. $(0: 02: 47-0: 03: 31)$
}

Die Beschreibung ist sachlich und neutral. Die einzigen Wörter, die als Schlüsselworte fungieren und somit adäquate Schemata und die damit verbunden Vorstellungsinhalte beim Zuschauer aufrufen können, sind Substantive und Adjektive, die auf die Vergitterung des Gebäudes hinweisen. Wie zu erkennen, bilden fast ausschließlich eingliedrige Substantive die Darstellung dieses Handlungsraumes. Selten werden sie von Adjektiven bzw. Attribuierungen begleitet: einen weiten Hof, über niedrigen weißen Gebäuden. 
Im Vergleich zur polnischen Beschreibung scheint die deutsche Darstellung des Hofes detaillierter zu sein. Marciniak und Szymańska präzisieren in der ersten Beschreibung das Aussehen des Hofes nicht. Substantiv Terrakottaziegel und Adjektive wie weit, niedrig, weiß kommen nur in der deutschen Version vor. Man weist aber auch die Vergitterung des Gebäudes hin:

Mężczyzna w czarnej skórzanej kurtce i ciemnych okularach. Przechodzi przez bramę na dziedziniec. Gołębie zrywaja się do lotu. Na luku brwiowym mężczyzny plaster.

Na twarzy lekki zarost. Unosi głowę. W oknach kraty. Kolejno otwieraja się okna. Zza krat wygladają dzieci w różnym wieku. Mężczyzna przechodzi lukowato sklepionym wejściem. Wewnątrz mrok ostania jego twarz.

Manche von den erwähnten Unterschieden kompensiert die polnische Fassung an weiteren Stellen des Films. Es erscheint die Information, dass der Hof weit ist Widok na rozległy dziedziniec.

In der polnischen Beschreibung betont man an mehreren Stellen, dass der Hof sich hinter der Mauer befindet: Naprzeciw mur z wejściem, $W$ murze po prawej uchylone drzwi, Mężczyzna idzie w stronę muru, Za murem oświetlone miejskie zabudowania, Z lewej mur z brama, dwukondygnacyjny budynek, buda, schody prowadzace w lewo. Die vermittelten Details ermöglichen es, die Topographie des Hofs wiederzugeben und verstärken das Gefühl der Enge und Abriegelung der Klinik.

In den beiden Fassungen des Hörfilms Imagine sind Beispiele für die Beschreibung von Handlungsräumen zu finden, die über eine neutrale Benennung von Orten und Gegenständen hinausgehen und für die Wiedergabe der filmischen Poetik von Bedeutung sind. Es werden bewusst Sachverhalte erwähnt, die die Eindrücke der Rezipienten in eine bestimmte Richtung lenken und die Stimmung des Augenblicks wiedergeben. Sowohl in der deutschen als auch in der polnischen Version beziehen sie sich auf das Licht in der Nacht: der Hof im kalten Licht einer Laterne, dziedziniec pograżony w mroku, und auf das Tageslicht: der Hof ist sonnendurchflutet, dziedziniec zatopiony w stońcu.

Der Eindruck der Enge in Imagine wird zusätzlich dadurch verstärkt, dass die Handlung im ersten Akt entweder im Hof - na dziedzińcu, im Gang - na korytarzu oder im Zimmer des Lehrers (in der polnischen Fassung nicht benannt) und in einem Unterrichtsraum - sala spielt. Wie zu erkennen, stellen fast ausschließlich Substantive die erwähnten Handlungsräume dar. Ein Adjektiv kommt mit schlicht (in einem schlichten Zimmer) nur in der deutschen Fassung vor. Sachlichkeit und Neutralität der Beschreibung wird auch sowohl im Deutschen als auch im Polnischen durch die sparsame Verwendung von Synonymen und Unter- bzw. Oberbegriffen hervorgehoben. Die direkte, wörtliche Wiederaufnahme von Begriffen dient der schnellerer Orientierung des Rezipienten und erleichtert das Wiedererkennen, andererseits wird so, wie Anja Seiffert (2005: 74) zu Recht bemerkt, eine Möglichkeit vergeben, Be- 
deutungen zu differenzieren und zusätzliche semantische Merkmale in den Text einzubringen. Dies bezieht sich auch auf das konnotative Potential des polnischen und deutschen Deskriptionstextes.

\section{Resümee}

Durch die Einführung des Verfahrens der Audiodeskription wird eine gesellschaftliche Ausgrenzung sehbehinderter Menschen von den Kulturgütern Fernsehen, Kino, Theater oder Museum verhindert. Somit können sie den Anforderungen der Mediengesellschaft gerecht werden und gleichwertig wie unbehindert Sehende am kulturellen Leben teilhaben. Die Übersetzung der Audiodeskription von einer Sprache in eine andere ist keine gängige Praxis, aber die Auffassung der Audiodeskription in den Kategorien einer sozialen und kulturellen Integrationshilfe und der damit verbundene wachsende Bedarf an Audiodeskriptionsfassungen lässt es notwendig erscheinen, das Thema der Manuskriptübersetzung zur Diskussion zur stellen.

Im Rahmen des vorliegenden Beitrags wurde der Versuch unternommen, durch eine kritische Revision der in der Filmbeschreibung geltenden Qualitätsstandards sowie der in Deutschland und Polen üblichen Konventionen der Textsorte Audiodeskription die Grundlagen für eine solche Diskussion zu liefern. In Anbetracht des beschränkten Umfangs des Beitrags wurde allein auf die Problematik der Beschreibung der handelnden Personen und der Handlungsräume eingegangen. Aus diesem Grund muss hervorgehoben werden, dass diese Arbeit keinen Anspruch auf Allgemeingültigkeit erhebt, vielmehr versteht sie sich als Anstoß zu weiteren Untersuchungen auf diesem Gebiet.

Durch die kontrastive Analyse konnte verdeutlicht werden, an welchen Stellen von den polnischen und deutschen Filmbeschreibern ähnliche und an welchen Stellen unterschiedliche Vorgehensweisen gewählt wurden. Die Untersuchung zeigt, dass die polnischen und die deutschen Konventionen zu Textgestaltung weitgehend übereinstimmen und die Idee der Audiodeskriptionsübersetzung umsetzen. Beide Fassungen sind von einfachen Sätzen und Satzstrukturen geprägt. Sie folgen auch ähnlichen Regeln in den Personenkennzeichnungen und der Beschreibung von Handlungsräumen. In beiden Audiodeskriptionsfassungen sind die Beschreibungen nicht mit Informationen überladen, man lässt dem Publikum stumme Pausen, die das Verarbeiten des Gehörten ermöglichen. Die Filmdialoge werden nicht übersprochen und auch für die Poetik des Films so wichtiger Musik und Hintergrundgeräuschen wird genügend Platz eingeräumt. Der Grund, weshalb in beiden Fassungen an unterschiedlichen Textstellen bestimmte Details des besprochenen Handlungsraumes und der agierenden Figuren aufgegriffen werden, ist wahrscheinlich teilweise in der Sprachökonomie, in erster Linie jedoch der subjektiven Wahrnehmung der Filmbeschreiber zu suchen. Aus der Vielfalt von Informationen auf der Leinwand können 
in der Audiodeskription nicht alle berücksichtigt werden, es muss eine Auswahl stattfinden. Der Filmbeschreiber muss die Entscheidung treffen, in welche der Lücken zwischen den Toninformationen er eine ausgewählte Bildinformation einfügen soll. Dabei ist sowohl der technisch zur Verfügung stehende Raum als auch das Herstellen einer sinnvollen Ganzheit mit Dialogen, Geräuschen und Musik zu berücksichtigen. Aus der Analyse der polnisch- und deutschsprachigen Version des Hörfilms Imagine ergibt sich, dass es beiden Audiodeskriptionsfassungen gelingt, den blinden und sehbehinderten Rezipienten die Handlung und Stimmung des Films adäquat zu vermitteln.

\section{Literatur}

Audiodeskrypcja do filmów zagranicznych, in: <http://avt.ils.uw.edu.pl/ad-foreign/>, (Zugriff am 15.10.2014).

AVT Lab Audiovisual Translation Research Lab - <http://avt.ils.uw.edu.pl/>, (Zugriff am 15.10.2014).

Benecke, Bernd: Audiodeskription als partielle Translation. Modell und Methode, Berlin 2014.

Benecke, Bernd, Dosch, Elmar: Wenn aus Bildern Worte werden - Durch Audio-Description zum Hörfilm, München2004.

Bittner, Hansjörg: Audio Description Guidelines - a Comparison, in: New Perspectives in Translation 2012, 20, S. 41-61.

Bogucki, Łukasz: Ares and Methods of Audiovisual Translation Research, Frankfurt am Main 2013.

Bourne, Julian, Hurtado, Catalina Jiménez: From the visual to the verbal in two languages: a kontrastive analysis of the audio description of The Hours in English and Spanish, in: Cintas Jorge Díaz, Orero Pilar, Remael Aline (Hrsg.): Media for All. Subtitling for the Deaf, Audio Description, and Sign Language, Amsterdam/New York 2007, S. 175-187.

Chmiel, Agnieszka, Mazur, Iwona: Overcoming barriers - The pioneering years of audiodescription in Poland, in: Şerban Adriana, Matamala Anna, Lavaur Jean-Marc (Hrsg.): Audiovisual translation in close-up. Practical and Theoretical Approaches, Bern 2011, 2012, S. 279-296.

Chmiel Agnieszka, Mazur, Iwona: Percepcja filmu a ogólnoeuropejskie standardy audiodeskrypcji polski wkład w projekt „Pear Tree”, in: Przekładaniec. O przekładzie audiowizualnym 2008, 1, S. $138-158$.

Dedecius, Karl: Vom Übersetzen. Theorie und Praxis, Frankfurt am Main 1986.

Díaz Cintas, Jorge, Orero, Pilar, Remael, Aline: Media for all: A global challenge, in: Díaz Cintas Jorge, Orero Pilar, Remael Aline (Hrsg.): Media for all. Subtitling for the Deaf, Audio Description, and Sign Language, Amsterdam/New York 2007, S. 11-20.

Fix, Ulla: Bild wahrnehmen, ohne zu sehen? Bildlichkeit in der Audiodeskription von Hörfilmen, in: Diekmannshenke Hajo, Klemm Michael, Stöckl Hartmut (Hrsg.): Bildlinguistik: TheorienMethoden-Fallbeispiele, Berlin 2011, S. 305-329.

Fix, Ulla (Hrsg.): Hörfilm. Bildkompensation durch Sprache. Linguistisch-filmisch-semiotische Untersuchungen zur Leistung der Audiodeskription in Hörfilmen am Beispiel des Films „Laura, mein Engel“ aus der „Tatort-Reihe“, Berlin 2005.

Fundacja Audiodeskrypcja [Stiftung Audiodeskription] - <www.audiodeskrypcja.org.pl >, (Zugriff am 15.10.2014).

Fundacja Kultury bez Barier [Stiftung Kulturen ohne Barrieren] - <www.kulturabezbarier.org>, (Zugriff am 15.10.2014). 
Hyks, Veronika: Audio Description and Translation: Two Related but Different Skills, in: Translating Today 2005, 4, S. $6-8$.

Jankowska, Anna 2014: Ttumaczenie jako alternatywna metoda tworzenia audiodeskrypcji, in: Przekładaniec. Audiodeskrypcja 2014, 28, S. 23-38.

Kluckhohn, Kim 2005: Infromationsstrukturierung als Kompensationsstrategie- Audiodeskription und Syntax, in: Fix Ulla (Hrsg.): Hörfilm. Bildkompensation durch Sprache. Linguistisch-filmischsemiotische Untersuchungen zur Leistung der Audiodeskription in Hörfilmen am Beispiel des Films „Laura, mein Engel“" aus der „,Tatort-Reihe“, Berlin, S. 49-65.

Korycińska-Wegner, Małgorzata: Übersetzer der bewegten Bilder. Audiovisuelle Übersetzung - ein neuer Ansatz, Frankfurt am Main 2011.

Krysztofiak, Maria: Einführung in die Übersetzungskultur, Frankfurt am Main 2013.

Künstler, Izabela: Audiodeskrypcja - jak to się robi?, in: Więckowski Robert, Raczek Tomasz, Künstler Izabela: Biała laska po kinowym ekranie, Warszawa 2010, S. 14-20.

Künstler, Izabela: Cel uświęca środki audiodeskrypcji, in: Przekładaniec. O przekładzie audiowizualnym 2008, 20, S. 140-152.

Künstler, Izabela, Butkiewicz, Urszula, Więckowski, Robert: Audiodeskrypcja zasady tworzenia. Dokument für die Stiftung Kultury bez barier bearbeitet 2012, in: <http://kulturabezbarier.org/contai ner/Publi kacja/Audiodeskrypcja\%20\%20zasady\%20tworzenia.pdf>, (Zugriff am 15.10.2014).

Masłowska, Karolina: Audiowstęp jako sposób na uzupetnienie audiodeskrypcji, in: Przekładaniec. Audiodeskrypcja 2014, 28, S. 39-47.

Maszerowska, Anna, Matamala, Anna, Orero, Pilar (Hrsg.): Audio Description New perspectives illustrated, Amsterdam/Philadelphia 2014.

Michalewicz, Irena: Audiodeskrypcja po Euro 2012 - zawrotne tempo akcji czy para $w$ gwizdek?, in: Przekładaniec. Audiodeskrypcja 2008, 20, S. 153-162.

Offizielle deutschsprachige Website des Films Imagine - <www.imagine-der-film.de>, (Zugriff am 15.10.2014)

Puigdomènech, Laura, Matamala, Anna, Orero, Pilar: Audio description of films: state of the art and protocol proposal, in: Bogucki Łukasz, Kredens Krzysztof (Hrsg.): Perspectives on Audiovisual Translation, Frankfurt am Main 2010, S. 27-43.

Seiffert, Anja 2011: Räumliches hören. Eine schemaorientierte Analyse der audiodeskriptiven Darstellung der Handlungsräume, in: Fix Ulla: Bild wahrnehmen, ohne zu sehen? Bildlichkeit in der Audiodeskription von Hörfilmen, in: Diekmannshenke Hajo, Klemm Michael, Stöck1 Hartmut (Hrsg.): Bildlinguistik: Theorien-Methoden-Fallbeispiele, Berlin, S. 67-86.

Stöckl, Hartmut: Sprache-Bild-Texte lesen. Bausteine zur Methodik einer Grundkompetenz, in: Diekmannshenke Hajo, Klemm Michael, Stöckl Hartmut (Hrsg.): Bildlinguistik: Theorien-MethodenFallbeispiele, Berlin 2011, S. 45-70.

Szarkowska, Agnieszka: Audiodeskrypcja oczami niewidomych - wywiad z Tomaszem Strzymińskim, in: Przekładaniec. O przekładzie audiowizualnym 2008, 1, S. 125-130.

Szarkowska, Agnieszka: Audiodeskryberem być. Wywiad z Krzysztofem Szubzda, pierwszym audiodeskryberem w Polsce, in: Przekładaniec 2008, 20, S. 130-135.

Szarkowska, Agnieszka, Wasylczyk, Piotr: Audiodeskrypcja autorska, in: Przekładaniec. O przekładzie audiowizualnym 2008, 20, S. 48-62.

Szymańska, Barbara, Strzymiński, Tomasz: Standardy tworzenia audiodeskrypcji do produkcji audiowizualnych, in: <http://www.audiodeskrypcja.org.pl/standardy-tworzenia-audiodeskrypcji/do-produkcjiaudiowizualnych.html?showall=1\&limitstart=>, (Zugriff am 15.10.2014).

Vercauteren, Gert: Towards a Eurpean guideline for audio description, in: Cintas Jorge Díaz, Orero Pilar, Remael Aline (Hrsg.): Media for All. Subtitling for the Deaf, Audio Description, and Sign Language, Amsterdam/New York 2007, S. 139-149. 
Vereinigung Deutscher Filmbeschreiber $-<$ www.hoerfilmev.de $>$, (Zugriff am 15.10.2014).

Weißbach, Marleen: Audiodeskription und Hörfilme. Eine kontrastive Analyse der deutschen und englischen Audiodeskription des Film „Brokeback Mountain “, in: Panier Anne, Brons Kathleen, Wisniewski Annika, Weißbach Marleen: Filmübersetzung. Probleme bei Synchronisation, Untertitelung, Audiodeskription, Frankfurt am Main 2012, S. 343-409.

Więckowski, Robert: Na imię ma Audiodeskrypcja, in: Więckowski Robert, Raczek Tomasz, Künstler Izabela: Biała laska po kinowym ekranie, Warszawa 2010, S. 5-9.

Więckowski, Robert, Raczek, Tomasz, Künstler, Izabela: Biała laska po kinowym ekranie, Warszawa 2010. 